This item was submitted to Loughborough's Research Repository by the author.

Items in Figshare are protected by copyright, with all rights reserved, unless otherwise indicated.

\title{
Modeling evaporation, ion-beam assist, and magnetron sputtering of thin metal films over realistic time scales
}

PLEASE CITE THE PUBLISHED VERSION

http://dx.doi.org/10.1103/PhysRevB.86.035416

PUBLISHER

(C) American Physical Society

VERSION

VoR (Version of Record)

LICENCE

CC BY-NC-ND 4.0

\section{REPOSITORY RECORD}

Blackwell, Sabrina, Roger Smith, Steven D. Kenny, and Michael Walls. 2019. "Modeling Evaporation, Ionbeam Assist, and Magnetron Sputtering of Thin Metal Films over Realistic Time Scales". figshare. https://hdl.handle.net/2134/11614. 
This item was submitted to Loughborough's Institutional Repository (https://dspace.lboro.ac.uk/) by the author and is made available under the following Creative Commons Licence conditions.

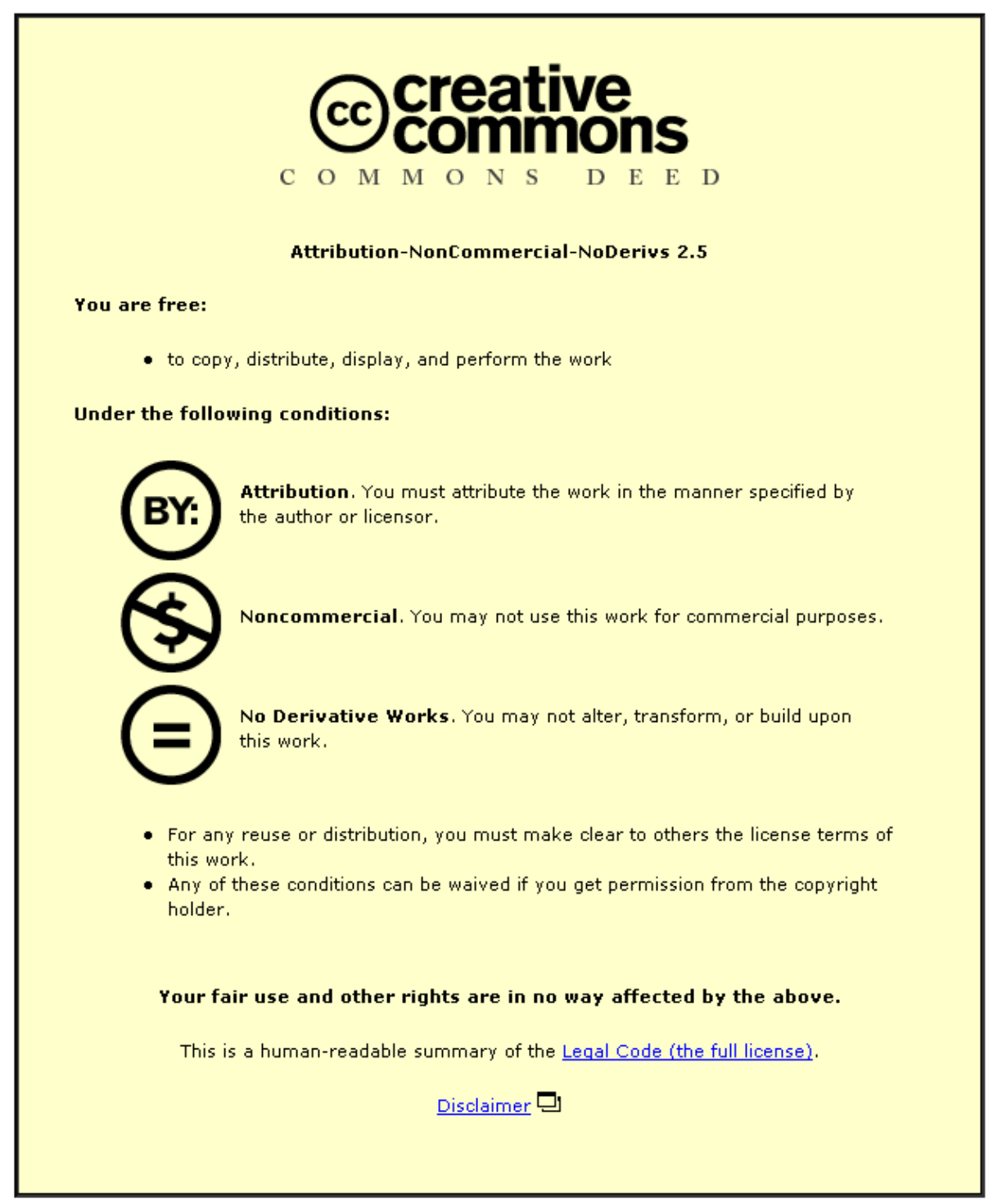

For the full text of this licence, please go to: http://creativecommons.org/licenses/by-nc-nd/2.5/ 


\title{
Modelling Evaporation, Ion-Beam Assist and Magnetron Sputtering of Thin Metal Films over Realistic Timescales
}

\author{
S. Blackwell, R. Smith, S. D. Kenny \\ Department of Mathematical Sciences, Loughborough University, Loughborough, Leicestershire, LE11 $3 T U$ \\ J. M. Walls \\ Department of Electronic and Electrical Engineering, \\ Loughborough University, Loughborough, Leicestershire, LE11 $3 T U$
}

(Dated: July 18, 2012)

\begin{abstract}
A long time scale dynamics technique has been used to model the evaporation, ion-beam assist and magnetron sputtering of thin metal films over realistic time scales. Two fcc metals have been investigated; silver and aluminium. We illustrate how the technique can be used to model growth of these films over experimental time scales, while investigating individual growth mechanisms and surface diffusion events. Long time dynamics is achieved through an on-the-fly Kinetic Monte Carlo method, which determines diffusion pathways and barriers, in parallel, with no prior knowledge of the involved transitions. It was found that $\mathrm{Ag}$ has the ability to grow smooth surfaces, using several mechanisms including multiple atom concerted motion, exchange mechanisms and damage and repair systems. Ag $\{111\}$ and $\{100\}$ grew dense, complete and crystalline film when sputtering was simulated, whereas evaporation produced incomplete layers. The inclusion of Ar in the ionbeam assisted evaporation of $\mathrm{Ag}\{111\}$ aided growth by transferring more energy to the surface atoms allowing increased diffusion. $\mathrm{Al}\{111\}$ however shows slightly different patterns; growth via evaporation and magnetron sputtering shows only slight differences and the inclusion of the ionbeam assist actually damages the film beyond repair producing subsurface Ar clusters where $\mathrm{Al}$ atoms were displaced creating voids throughout the film. Al $\{100\}$, similar to $\mathrm{Ag}\{100\}$ grows denser and more complete film when grown via sputtering rather than evaporation. Results show that the energy of the deposition method used, plays a vital role in the resulting thin film and substrate quality.
\end{abstract}

\section{INTRODUCTION}

Thin films of metals such as $\mathrm{Ag}$ and $\mathrm{Al}$ have important industrial applications based on their optical and electrical properties. The work described here has been predominantly motivated by the application of these metals in photovoltaic industry applications. For example these metals are used as reflectors in the optics used in Concentrator Photovoltaics $(\mathrm{CPV})^{1-3}$. They are also used as electrical conductors in the monolithic interconnect processes for thin film photovoltaics (a$\mathrm{Si}, \mathrm{CdTe}$ and CIGS). They are also used as back contacts.

Metal thin films can be deposited using a variety of industrial-scale processes, including evaporation (thermal and electron beam $)^{4}$, ion-beam assisted evaporation ${ }^{5}$ and magnetron sputtering ${ }^{6}$, all of which are physical vapour deposition (PVD) processes ${ }^{7}$. The evaporation process involves evaporation of either $\mathrm{Ag}$ or $\mathrm{Al}$ atoms onto the substrate with kinetic energy typically $<1 \mathrm{eV}$. A high energy ion source (usually Ar) may be used to densify the film; for example a $100 \mathrm{eV}$ ion-beam introduces extra energy into the growing film ${ }^{5}$ to enhance mixing. Magnetron sputtering, either RF, DC or pulsed DC power, deposits material via sputtering of a metallic target by a working gas (Ar) within the magnetron plasma, providing atoms with $\sim 40 \mathrm{eV}$ of kinetic energy. Ar in the plasma also bombard the substrate with a similar kinetic energy thus affecting the density and stoichiometry of the growing film ${ }^{5,8,9}$. Experimental evidence exists to confirm that during both deposition processes the Ar presence plays a role in the growth quality; Ar bombardment has been shown to improve the density of films due to the bombardment transferring momentum to the substrate thus enhancing surface mobility. However, literature also shows that in $\mathrm{Al}$ thin films high energy Ar ion bombardment from the ion-beam can damage substrates by the formation of subsurface Ar agglomeration ${ }^{10-13}$.

Previously, researchers have modelled thin film growth and specifically modelled the different deposition processes used in the growth of numerous different materials ${ }^{14-17}$. Theoretical and experimental work has been carried out in the past on the thin film behaviour of $\mathrm{Ag}$ and $\mathrm{Al}$. Research has been undertaken on surface diffusion of both $\mathrm{Al}$ and $\mathrm{Ag}$ on various surfaces using classical MD, temperature accelerated dynamics (TAD) and other long time scale dynamics techniques ${ }^{18-23}$. Studies using just typically MD methods only allow simulation of systems for a few hundred picoseconds, which does not allow for realistic modelling of thin film growth where the processes occur over longer time scales. TAD methods allow simulation over much longer timescales by increasing temperature to accelerate the dynamics of a system and then correcting to the relevant temperature. The long time scale techniques employed in this work calculate the transition paths by direct 
climbing to the saddle points, allowing deeper and more accurate investigation of $\mathrm{Ag}$ and $\mathrm{Al}$ thin film behaviour by including diffusion events between impacts. Long time scale techniques allow growth to be simulated for seconds or longer, which is unattainable using the traditional MD methods.

This work investigates, at the atomistic level, the effect of the different deposition processes introduced above on the layer by layer growth of $\mathrm{Ag}$ and $\mathrm{Al}$. Focus is placed on the mechanisms by which growth occurs and the difference between the quality of growth produced under different deposition conditions. The effect of Ar involvement either through direct bombardment as with an ion beam or through its natural inclusion in the plasma of a typical magnetron device is also investigated.

\section{METHODOLOGY}

Atomistic simulation has been used as a complementary partner with experiment to address problems in materials science. Despite advances in computational power, using traditional molecular dynamics (MD) alone to model large systems over long time scales is computationally unfeasible. Traditional MD allows simulation of at most a few microseconds, depending on the system size, whereas the time required to grow one monolayer of $\mathrm{Ag}$ is at least several hundred milliseconds. Long time scale dynamics combines MD with new techniques, allowing simulation over experimental time scales without biasing the system dynamics ${ }^{25}$. Here, time scales are extended using on-the-fly Kinetic Monte Carlo (otf-KMC) ${ }^{26-28}$. Combining MD with this otf-KMC method allows simulation of systems over several seconds, allowing the simulation of growth of several monolayers.

Simulations were carried out using the Ackland EAM potential for $\mathrm{Ag}^{29}$ and the Voter and Chen potential for $\mathrm{Al}^{30}$. When Ar was included in the model a Lennard Jones potential modelled the interaction between $\mathrm{Ar}$ atoms $^{31}$ and the ZBL potential simulated the interaction between both $\mathrm{Ar}$ and $\mathrm{Ag}$ and $\mathrm{Ar}$ and $\mathrm{Al}^{32}$. $\mathrm{Pe}-$ riodic boundary conditions were employed for the substrate. Typical system sizes were 4-8 monolayers deep with between 128 and 225 atoms per monolayer. When simulating growth, we began with the appropriate Ag or $\mathrm{Al}$ surface with a trimer placed on top. In the absence of surface defects monomers and dimers were found to be highly mobile on both the $\mathrm{Ag}$ and $\mathrm{Al}$ surfaces but can gradually combine into clusters which become less mobile, therefore we began simulations with a trimer on the surface which acted as a nucleation site for the growth thus saving computational time at the start of the simulation. Either an $\mathrm{Ag}$ or $\mathrm{Al}$ atom, respectively, was deposited onto the surface with the desired kinetic energy by first running MD between 4 and 10 ps. The system was then relaxed and diffusion between deposition events was simulated using otf-KMC. At each step either a deposition event or a diffusion event is chosen, according to their relative probabilities. Growth continued in this way until enough monolayers have been deposited. Here, three monolayers of atoms were added. Note that the growth technique involves MD and otf-KMC working together in parallel over typically $48 \mathrm{cpu}$ cores, where the deposition event runs MD on a single core and searches are employed on multiple cores. Figure 1 demonstrates this client/server model. The methodology is further described below.

\section{A. Molecular Dynamics}

During the MD stage, the bottom layer of the lattice was fixed and a Berensden ${ }^{33}$ thermostat was attached to the next two monolayers to remove any excess energy from the system caused by the deposition. Randomly oriented atoms were deposited normally to the surface, with the deposition energies selected from a normal distribution with a standard deviation a tenth of the deposition energy (specific to each simulation). The lattice was heated to just above room temperature $(350 \mathrm{~K})$ before each deposition and MD then ran until the lattice returned to this starting temperature and defects had stabilised, taking typically 4 -10 ps, depending on deposition energy used. After this time the system was relaxed before the transition searches were undertaken.

\section{B. On-the-fly Kinetic Monte Carlo}

Traditional Kinetic Monte Carlo (KMC) involves predefinition of all available transitions. This is useful for simple, symmetric crystal structures, but as systems become more complex, traditional KMC cannot perform accurately. When dealing with highly defective systems, it becomes almost impossible to pre-define all the transitions. Otf-KMC aims to rectify this issue by calculating transitions on-the-fly. The otf-KMC algorithm involves four fundamental steps, described below:

1. Identification of all the defects by comparison to a perfect bulk lattice. This produces a defect lattice where defects and their neighbouring atoms are included. These are included in the search space for a transition.

2. Search for all possible transitions involving only the atoms defined in the search vector. Locate saddle points using the Relaxation and Translation method (RAT) ${ }^{24}$ and then use the climbing image Nudged Elastic Band method $(\mathrm{NEB})^{34-36}$ to determine barrier heights more accurately once the transition has been found. Typically $\sim 200$ searches are carried out per KMC step. 
Server

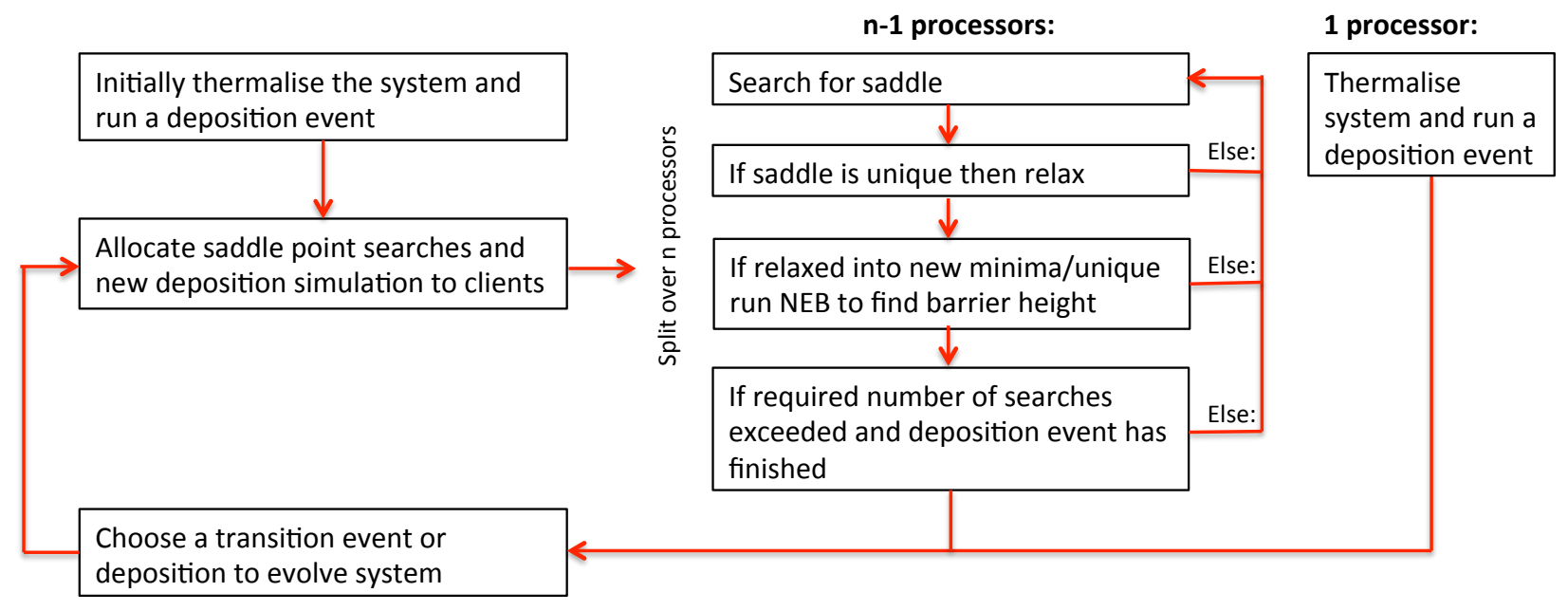

FIG. 1. The client/server model allowing parallelisation of transition searches, saddle relaxing and barrier height calculation.

3. Calculate transition rates of every unique transition found using the Arrhenius equation (1):

$$
\text { Escape Frequency }=\nu \exp \left(-E_{b} / k_{B} T\right)
$$

where $\nu$ is the transition prefactor, $E_{b}$ is transition barrier, $k_{B}$ is the Boltzmann constant and $T$ is the temperature (Kelvin). $\quad \nu$ can be calculated for each transition using the Vineyard ${ }^{37}$ method, however, due to computational time limitations, $10^{13} \mathrm{~s}^{-1}$ is taken to be the prefactor ${ }^{24}$.

4. Transition searches together with a deposition event are carried out in parallel as described previously. Either a transition or deposition event is chosen from a roulette algorithm, and is used to evolve the system in time.

\section{RESULTS}

\section{A. Deposition on the Ag $\{111\}$ Surface}

\section{Evaporation Deposition}

On the $\{111\}$ surface transition barriers for single adatoms and small clusters were initially calculated and are shown in table I. Single ad-atoms and smaller clusters are planar and require as little as $0.12 \mathrm{eV}$ to diffuse, whereas larger clusters required up to $0.45 \mathrm{eV}$. Bonding to a step edge of a single ad-atom required very little energy, however $0.74 \mathrm{eV}$ was required for the de-bonding. Figure 2 illustrates a four atom cluster diffusing during early stages of growth where the cluster diffuses between stacking sequences with an energy barrier of $0.4 \mathrm{eV}$. It is clear, however, that single ad-atom hops and step edge bonding requiring only $0.12 \mathrm{eV}$, should dominate the simulation.
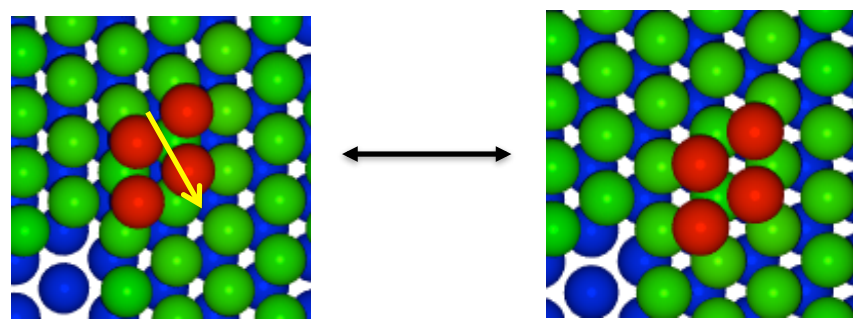

FIG. 2. (color online) A four atom cluster on the $\mathrm{Ag}\{111\}$ surface during early stages of evaporation growth switching between $\mathrm{ABC}$ and $\mathrm{ABAB}$ stacking sequences. $0.4 \mathrm{eV}$ is required for this transition, with only half of that energy required to switch back to the preferred $\mathrm{ABC}$ sequence. Once a fifth atom attaches to the cluster it pins the cluster to the layer it is in. Atoms are coloured by height, with the cluster being in red.

Figure 3 illustrates the resulting growth of $\mathrm{Ag}\{111\}$ via evaporation deposition where $\mathrm{Ag}$ atoms arrive at the substrate with kinetic energy of typically $<1 \mathrm{eV}$. It is clear that layers are incomplete and holes are present in the film. 675 atoms (equivalent to three monolayers) have been deposited onto the substrate, but these atoms actually produce six new layers, all of which are incomplete. Due to atoms arriving at the substrate with such low kinetic energy, there is not enough energy for layers to self-complete via surface diffusion. No mixing occurs between original substrate and the newly grown material, illustrating again that atoms with low deposition energy tend to contact the surface and immediately stick. 


\begin{tabular}{cccccc}
\hline \hline Monomer hop & Dimer hop & Trimer rotation & Trimer hop & 4-mer hop & 5 -mer hop \\
\hline $0.12 \mathrm{eV}$ & $0.22 \mathrm{eV}$ & $0.23 \mathrm{eV}$ & $0.28 \mathrm{eV}$ & $0.40 \mathrm{eV} / 0.26 \mathrm{eV}$ & $0.45 \mathrm{eV}$ \\
\hline \hline \multicolumn{5}{c}{} \\
\hline \hline Bond to step edge & De-bond from step edge \\
\hline $0.12 \mathrm{eV}$ & $0.74 \mathrm{eV}$ & \\
\hline \hline
\end{tabular}

TABLE I. Transition barriers for single ad-atom and small cluster diffusion on the Ag $\{111\}$ surface.

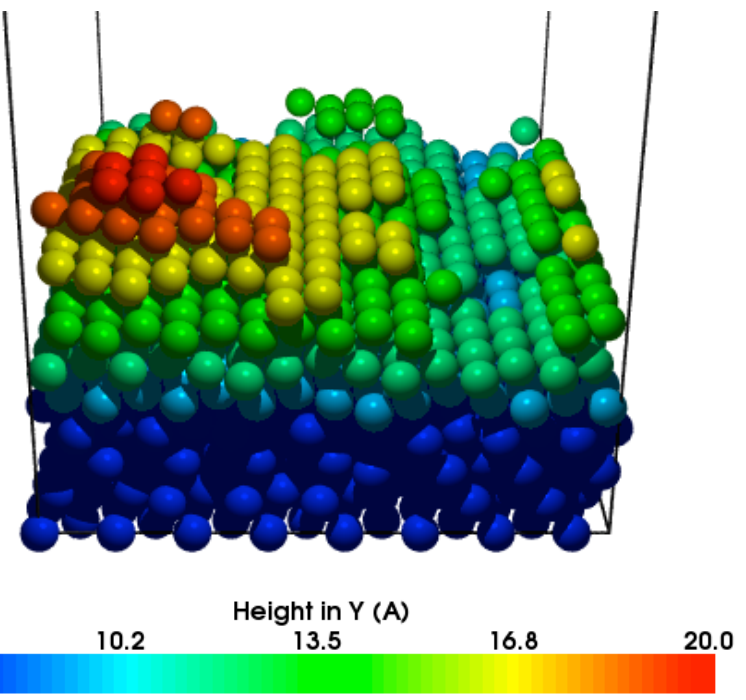

FIG. 3. (color online) Ag $\{111\}$ growth via evaporation deposition after $0.29 \mathrm{~s}$ of real time. 675 atoms have been added to the system, equivalent to three complete monolayers, corresponding to a growth rate of ten monolayers per second which is used for all simulations. Six partially formed layers have grown, although all are in the ABAB stacking sequence rather than the preferred ABC. Atoms are coloured by height in $\AA$, according to the colour bar, with the original surface at $7 \AA$.

\section{Stacking faults and twinning}

Previous experiments using low energy ion scattering to examine the first few layers grown via vapour deposition have shown that growth does not always occur in a completely crystalline manner ${ }^{38}$. It was found that $\mathrm{Ag}$ structures once grown can contain stacking faults and twin boundaries ${ }^{38}$. In this simulation, stacking faults in the film are observed and a mechanism for twinning would be having two five atom clusters on the surface both pinned in different positions and growing into a twin boundary. Ag $\{111\}$ is in a close packed structure, with ABC stacking sequence. It was calculated that a trimer on the $\{111\}$ surface diffuses from its stable $\mathrm{ABC}$ sequence into $\mathrm{ABAB}$ sequence with a relatively low energy barrier of $\sim 0.3 \mathrm{eV}$ (which has rate of $4.8 \mathrm{x}$ $10^{8} \mathrm{~s}^{-1}$, compared to deposition rate of $2.0 \times 10^{3} \mathrm{~s}^{-1}$ ). A cluster on the surface may switch between stacking sequences during the early stages of growth, however, when a cluster reaches 5 atoms it was observed that the cluster becomes pinned to the stacking sequence it is in. During the early stages of growth of the simulation shown in figure 3 , atoms sat in the $\mathrm{ABAB}$ sequence. The new layers were then pinned to this stacking sequence, thus all new layers grew in this manner, producing a stacking fault throughout the new film. The substrate, however, stayed in the original, correct stacking sequence.

\section{Deposition with Ion-Assisted Evaporation}

In experimental growth of thin films ion beam assist is often used to improve the quality of the crystalline growth. Here we model the process by MD. Ar ions/neutrals with $100 \mathrm{eV}$ of kinetic energy are assumed to bombard the surface at normal incidence. The Ar ion flux is assumed to be the same as that of the arriving Ag atoms, so that during the roulette process an Ar atom at $100 \mathrm{eV}$ or an $\mathrm{Ag}$ atom at $1 \mathrm{eV}$ is chosen with equal probability. The Ar ions help to densify the material ${ }^{5,8,9}$ and also promote increased surface atomic diffusion as the bombardments transfer kinetic energy to the system. Figure 4 shows the resulting growth when an Ar ion-beam assist is used along side the evaporation method; new layers sit in the correct stacking sequence, contrary to what was seen without the ion-beam assist. Due to the high impact energy of Ar, penetration deep into the the lattice was observed however by the end of the simulation all Ar had escaped from the substrate due to the low diffusion barriers. Previous otf-KMC simulations from literature have shown that residual Ar in Au films diffuses with very low energy barriers, many below $0.1 \mathrm{eV}^{28}$. Figure 5 illustrates the high portion of mixing occurring between atoms in the original substrate and the newly deposited atoms. Contrary to what was seen in the pure evaporation growth, here we see mixing due to the Ar bombardment, whereby original atoms are displaced, transferring kinetic energy to the surface.

By direct comparison of figures 3 and 4, we observe differences in film growth via purely evaporation and ion-beam assisted evaporation. Firstly, in the case of no ion-assist, six partially formed new layers are grown, whereas with the ion-assist only five partially formed layers are grown. This confirms that the ion-assist does aid the densification of the film through transferring kinetic energy to the atoms in the system enabling increased surface diffusion. Mixing is observed between atoms from the original substrate and the newly deposited atoms only when the ion- 


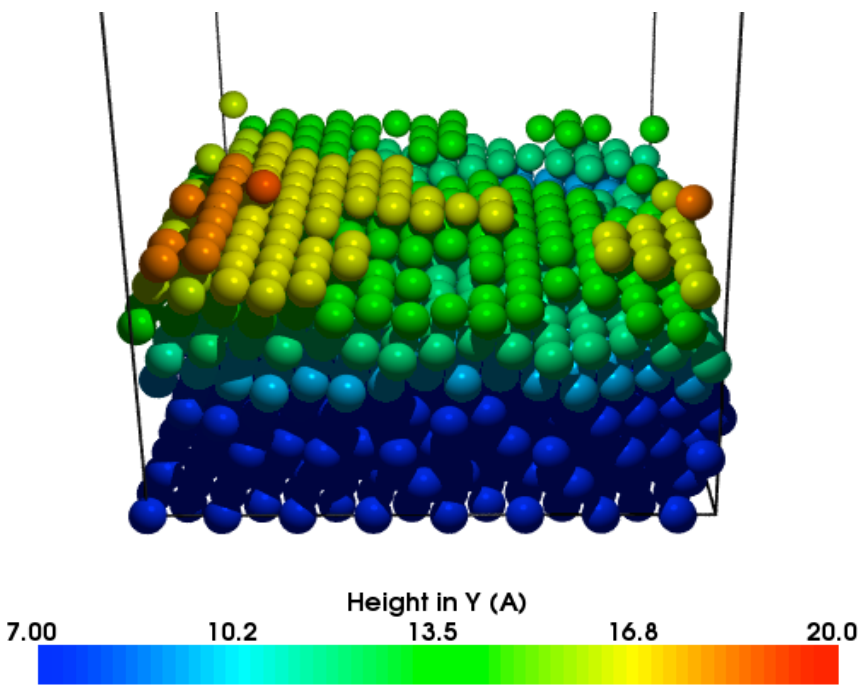

FIG. 4. (color online) Ag $\{111\}$ growth via evaporation deposition with ion-assist, where Ar ions bombard the surface with $100 \mathrm{eV}$ of kinetic energy, after $0.38 \mathrm{~s}$ of real time. Ar ions strike the surface with equal probability as an $\mathrm{Ag}$ atom. The extra energy which is transferred to the system from Ar bombardment promotes increased surface diffusion, leading to more complete layers. From the addition of 675 atoms, five partially formed monolayers have grown all in the correct ABC stacking sequences. Atoms are coloured by height in $\AA$, according to the colour bar.

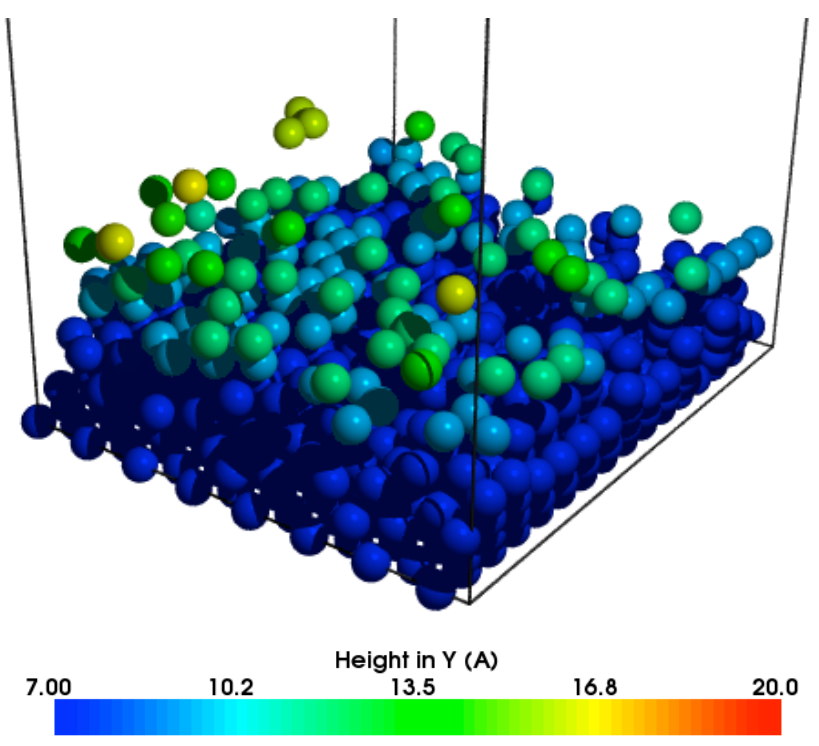

FIG. 5. (color online) Ag $\{111\}$ original substrate, showing atom positions after the simulation of evaporation deposition with the ion-beam assist. Atoms are coloured by height, where the height of the original substrate is at $7 \AA$. A high portion of original substrate atoms travel up to and above the original surface during the simulation, due to sputtering, displacement and mixing from high energy ion bombardments, which aid surface diffusion and thus growth. beam assist is included; otherwise no mixing at all occurs as the $1 \mathrm{eV} \mathrm{Ag}$ depositions do not transfer enough energy to displace any atoms from the substrate. Stacking faults are prominent in the film grown via evaporation; the film grows in ABAB stacking. Addition of the ion-beam assist provides enough kinetic energy to the film to eradicate stacking faults, thus the film grows in the preferred ABC manner.

\section{Sputter Deposition}

Sputter deposition is modelled by assuming that $\mathrm{Ag}$ atoms arrive randomly at the surface with an energy of $40 \mathrm{eV}$. In a magnetron sputtering device $\mathrm{Ar}$ is also present in the plasma. However in order to separate out the effects of Ar, two simulations have been performed. Figure 6 illustrates Ag growth excluding the effects of the Ar and figure 7 includes Ar. Again the Ar and Ag fluxes are assumed to be equal. The two simulations produce almost identical growth, with the first new layer being complete, and substantial mixing between atoms in the original substrate and added atoms allowing for subsequent layers to form almost complete. No stacking faults are observed due to the higher impact of arriving atoms transferring enough energy to the substrate to promote correct stacking. No Ar is retained within the film due to high Ar diffusion rates. Ar bombards the surface with $40 \mathrm{eV}$ so this is not enough energy to penetrate through the substrate therefore Ar tend to reflect off the surface.

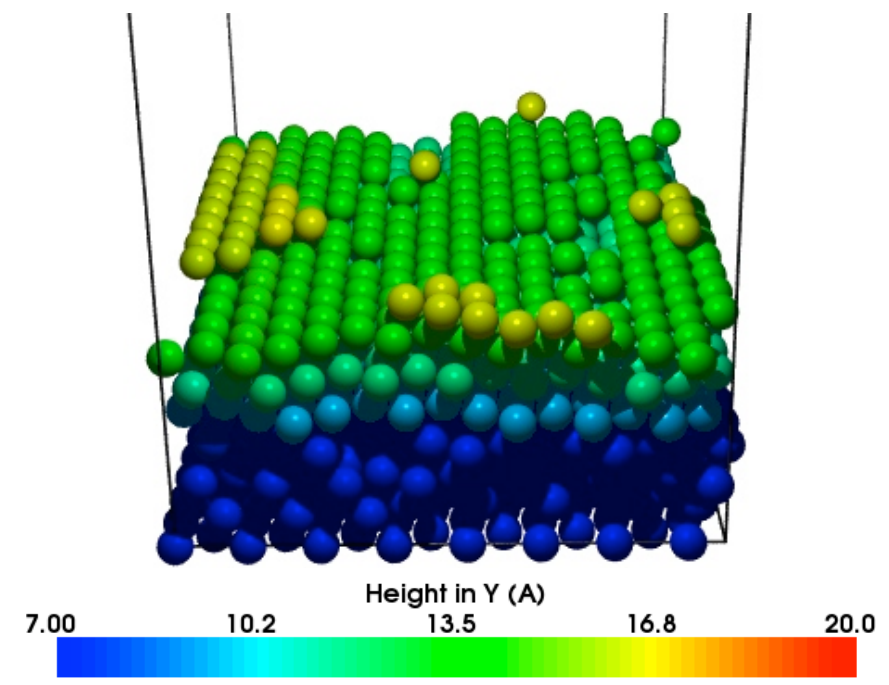

FIG. 6. (color online) $\mathrm{Ag}\{111\}$ growth from sputtering, after $0.27 \mathrm{~s}$ of real time. 675 atoms added to the system result in four newly formed layers, the first one of which is complete. Atoms are coloured by height in $\AA$, according to the colour bar.

Comparing the resulting growth of $\mathrm{Ag}\{111\}$ via evaporation, ion-beam assist and sputtering, it is clear 


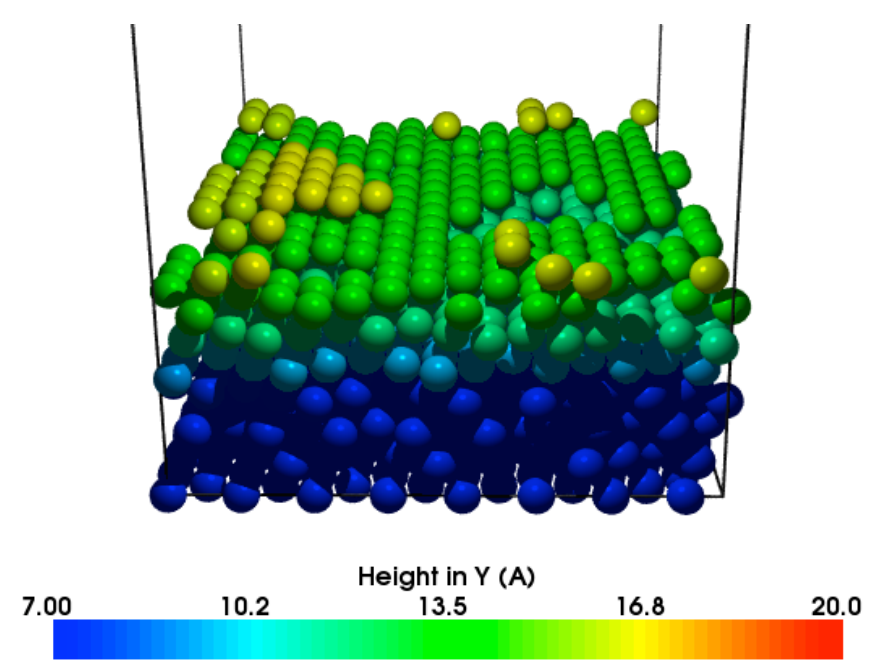

FIG. 7. (color online) Ag $\{111\}$ growth by magnetron sputtering, simulating $0.34 \mathrm{~s}$ of real time, including the effect of the Ar present in the plasma where there was equal likelihood that an $\mathrm{Ag}$ atom or an Ar atom would strike the surface. 675 atoms added results in four newly formed layers, similar to the simulation of sputtering alone. Atoms are coloured by height in $\AA$, according to the colour bar.

that evaporation deposition produces incomplete, void filled structures where stacking faults are prominent. The addition of the ion-beam introduces energy to the system allowing increased diffusion and slightly better crystallinity in the correct stacking sequence. Sputtering, with and without the inclusion of the Ar in the plasma, produces films that are denser and almost crystalline $^{5,8,9}$ and that sit in the correct ABC stacking.

Numerous growth mechanisms are observed during Ag $\{111\}$ growth; multiple atom concerted motions, exchange and replacement mechanisms, and vacancy filling mechanisms, all of which allow completion of monolayers and vacancies to be filled. The strength of the otf-KMC method is the ability to study in much detail these multiple atom transitions ${ }^{22}$. Multiple atom concerted motions are often observed, requiring only small energy barriers to be overcome. Figure 8 shows an example of two atoms which move in a concerted motion, sliding across to fill in a vacancy in the film with a energy barrier of $0.44 \mathrm{eV}$.

Figure 9 illustrates layer completion via the EhrlichSchwoebel (ES) barrier, a classic mechanism previously reported $^{39,40}$, where an atom drops off a step edge of an island in this case with a barrier of $0.42 \mathrm{eV}$. Transitions of these kinds enable films to grow in a complete, layer by layer manner, however as the ES barrier here is so much higher than a single ad-atom hop which requires only $0.12 \mathrm{eV}$, it will not occur many times in comparison to single atom hops, suggesting that cluster growth is more prominent here.

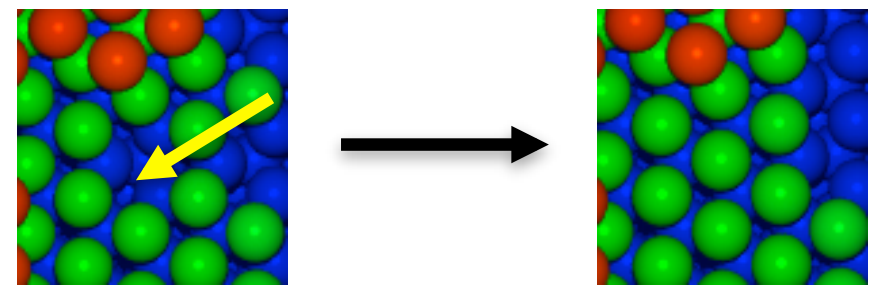

FIG. 8. (color online) Two atom concerted motion requiring $0.44 \mathrm{eV}$ of energy to take place. Ag $\{111\}$ film is able to self complete by filling in vacancies via this mechanism.
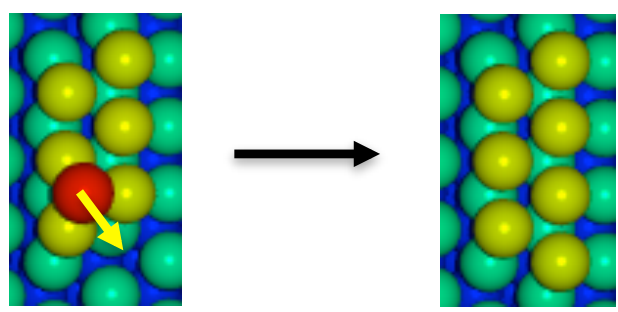

FIG. 9. (color online) The important ES transition requires $0.42 \mathrm{eV}$ to occur on the $\mathrm{Ag}\{111\}$ surface. This ES transition allows completion of layers, however as it is a higher energy barrier than that of the diffusion of a single ad-atom, it is not observed very frequently. Atoms are coloured according to height, where blue is the bottom, then green, then yellow and red.

\section{B. Deposition on the Ag $\{100\}$ Surface}

Simulations are now repeated on the $\{100\}$ surface. As previously we begin with a trimer on the surface to nucleate growth in order to save computational time during the early stages of growth.

\section{Evaporation Deposition}

Figure 10 shows resulting film growth from low energy evaporation depositions of single $\mathrm{Ag}$ atoms onto the $\operatorname{Ag}\{100\}$ substrate. Three complete monolayers were added to the system however it can be seen that new growth produces six new monolayers, of which none are complete. Vacancies are evident throughout the new film, which would lead to poor optical and electrical properties when used in photovoltaic devices. Evaporation as suggested earlier, involves atoms arriving at the surface with very little kinetic energy, hence on arrival at the surface very few atoms have the energy to diffuse into preferential locations. It is for this reason that highly incomplete monolayers are observed.

Growth shown in figure 11 is produced from our model of sputter depositions of Ag excluding the contribution of Ar from in the plasma. It can be observed that almost complete layers have formed as in the case of $\operatorname{Ag}\{111\}$. 

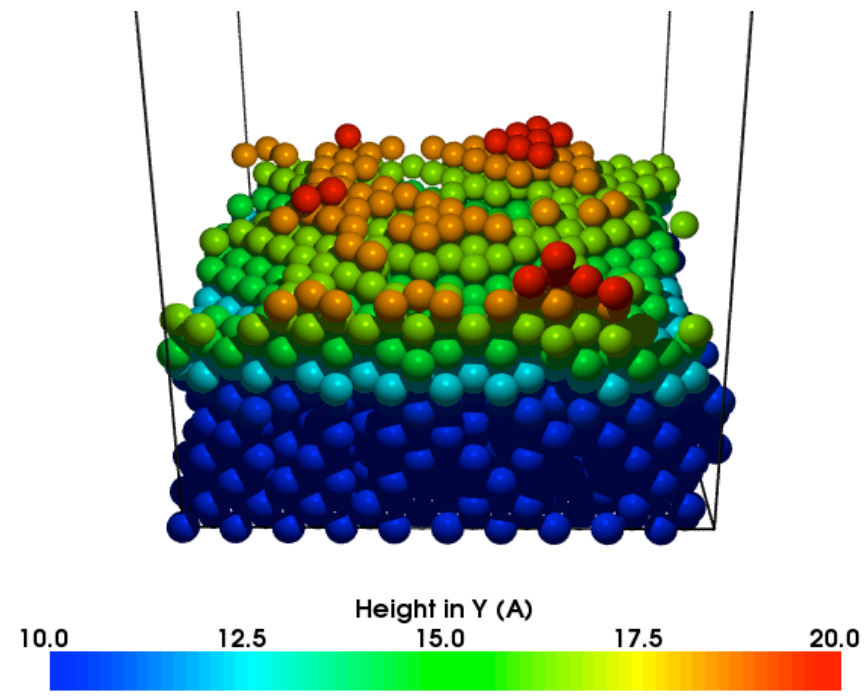

FIG. 10. (color online) $\mathrm{Ag}\{100\}$ growth via evaporation deposition after $0.23 \mathrm{~s}$ of real time, with the addition of 600 atoms (equivalent to three complete monolayers). Six incomplete monolayers are produced as evaporation does not transfer enough energy for surface diffusion thus layer completion to occur. The original substrate was at $10 \AA$.
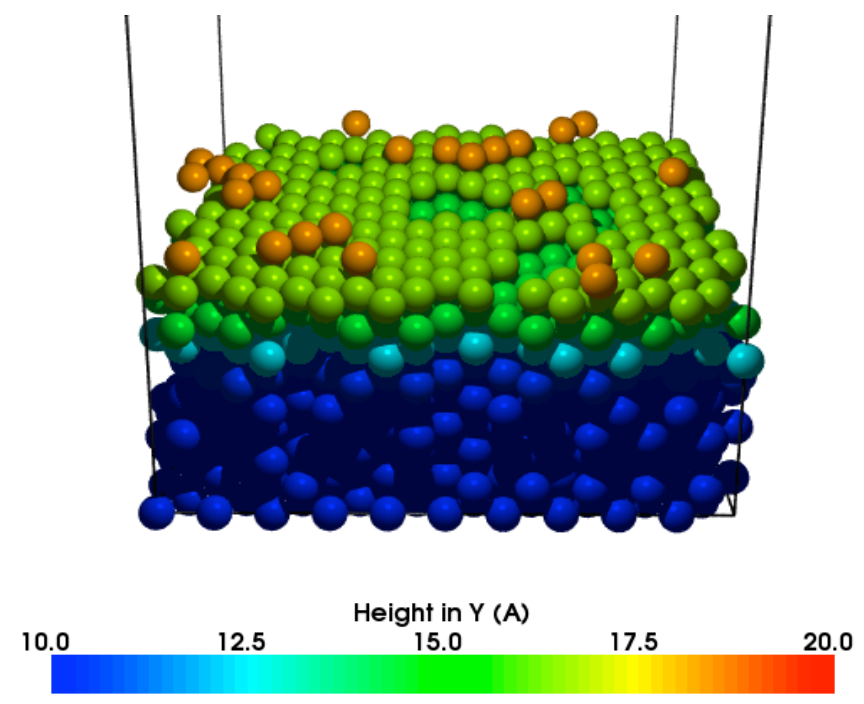

FIG. 11. (color online) Ag $\{100\}$ growth by magnetron sputtering, simulating $0.23 \mathrm{~s}$ of real time. The addition of 600 atoms produced three almost complete monolayers, along with a fourth incomplete monolayer just beginning.

When depositing on $\mathrm{Ag}\{100\}$, the calculated energy barriers were often greater than those seen with $\mathrm{Ag}$ $\{111\}$, typically ranging from $0.33 \mathrm{eV}$ to $0.65 \mathrm{eV}$. It was observed that the film grew via clusters, through linear islands forming on the surface and single $\mathrm{Ag}$ ad-atoms joining islands until they met and formed new monolayers. It was determined that a single $\mathrm{Ag}$ ad-atom transition on the surface required $0.65 \mathrm{eV}$ to diffuse, whereas cluster rearrangements and formation required as little as half of this energy, as depicted in the graph shown in figure 12, and listed in table II. Ag $\{100\}$ film therefore grows via many small clusters on the surface joining to form a layer rather than single atom hops as seen with the Ag $\{111\}$. The energy required for atoms to bond to step edges was actually less than required for a single monomer hop, with the reverse barrier only very slightly higher, agreeing with previous statements that cluster rearrangement often occurs.

The ES barrier was $0.42 \mathrm{eV}$ for this surface, the same observed with the $\{111\}$ surface. Figure 13 illustrates this ES transition and due to the high barrier this transition was not frequently observed. Film growth therefore occurred via separate clusters forming on the surface, rather than layer by layer growth. Due to the high ES barrier, atoms rarely dropped down down to complete lower layers, therefore causing clusters to grow in height rather than merge together, forming incomplete and somewhat rough surfaces.

\section{Deposition on the Al $\{111\}$ Surface}

The $\mathrm{Al}\{111\}$ substrate is investigated in the same way as $\mathrm{Ag}\{111\}$; evaporation deposition, ion-beam assist and magnetron sputtering growth are simulated. Small clusters on the surface are again mobile as they were on $\mathrm{Ag}$ $\{111\}$. For $\mathrm{Al}\{111\}$ even the 5 atom planar cluster has a small energy barrier for diffusion. This is show in figure 14 where it can move from $\mathrm{ABC}$ stacking to $\mathrm{ABAB}$ stacking with an energy barrier of $0.3 \mathrm{eV}$. Table III presents important transition barriers on the $\mathrm{Al}\{111\}$ surface.

\section{Deposition by Evaporation}

$\mathrm{Al}\{111\}$ grown by evaporation deposition is shown in figure 15. After the addition of three complete monolayers to the system it can be seen that two complete monolayers are produced, along with a third almost complete and the fourth has just begun. Despite the low kinetic energy of arriving atoms diffusion has occurred enabling such complete layers. This is contrary to the pattern seen with the $\mathrm{Ag}\{111\}$ where evaporation growth produced incomplete growth with voids. One explanation for this difference is that the energy required for a single ad-atom hop of $\mathrm{Ag}$ on the $\{111\}$ surface is between 0.1 and $0.15 \mathrm{eV}$, whereas for $\mathrm{Al}$ on the $\{111\}$ surface this is reduced to $<0.1 \mathrm{eV}$, suggesting that on $\mathrm{Al}\{111\}$ surface diffusion of single ad-atoms will occur more often which enables complete film growth to occur. A single $\mathrm{Ag}$ ad-atom hop on $\mathrm{Ag}\{111\}$ with a barrier of $0.15 \mathrm{eV}$ has a rate of $6.9 \times 10^{10} \mathrm{~s}^{-1}$ whereas an $\mathrm{Al}$ ad-atom hop requiring say $0.075 \mathrm{eV}$ has a rate of $8.3 \mathrm{x}$ $10^{11} \mathrm{~s}^{-1}$ which is over one order of magnitude more likely to occur. During growth, single $\mathrm{Al}$ ad-atoms diffuse freely over the surface with tiny energy barriers, joining 


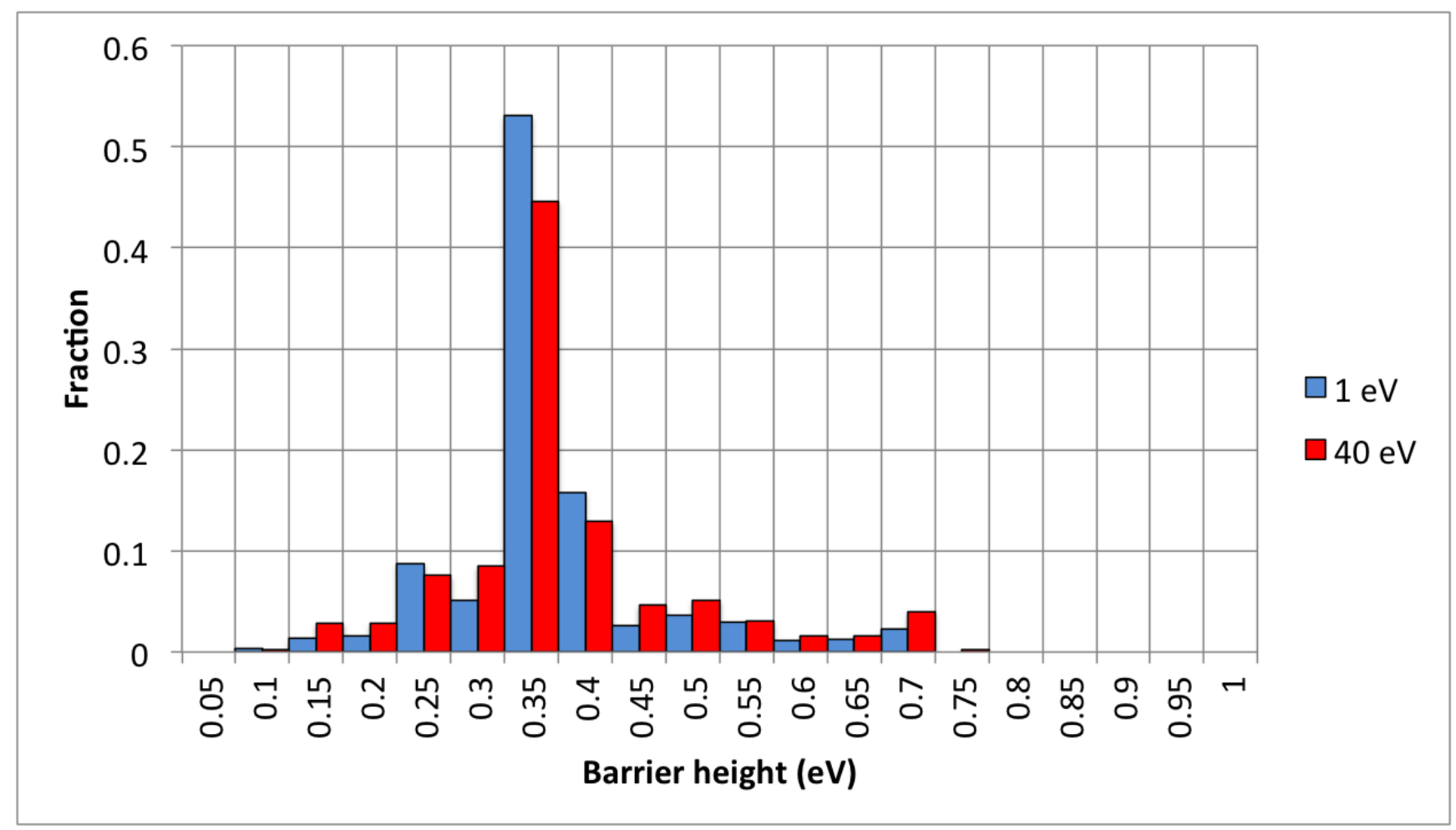

FIG. 12. (color online) The respective energy barrier heights for all those transitions observed during the Ag $\{100\}$ growth simulations. For both evaporation and sputter growth a sharp peak is observed at around $0.33 \mathrm{eV}$, which was found to be small cluster formations and rearrangements on the surface. Transitions requiring between $0.3 \mathrm{eV}$ and $0.4 \mathrm{eV}$ were shown to be larger cluster formation, rearrangement and addition to islands. Barriers around $0.41-0.42 \mathrm{eV}$ represent bonding and de-bonding from the step edge of single atoms. Single ad-atom hops required $0.65 \mathrm{eV}$ to take place; with such a large energy barrier this single ad-atom hop was rarely utilised (in less than $0.05 \%$ of transitions).

\begin{tabular}{ccc}
\hline \hline Monomer hop & Small cluster formation and rearrangement & Large cluster formation and rearrangement \\
\hline $0.65 \mathrm{eV}$ & $0.33 \mathrm{eV}$ & $0.3-0.4 \mathrm{eV}$ \\
\hline \hline & & \\
\hline \hline Bond to step edge & De-bond from step edge \\
\hline $0.41 \mathrm{eV}$ & $0.42 \mathrm{eV}$ \\
\hline \hline
\end{tabular}

TABLE II. Transition barriers for ad-cluster diffusion on the Ag $\{100\}$ surface.
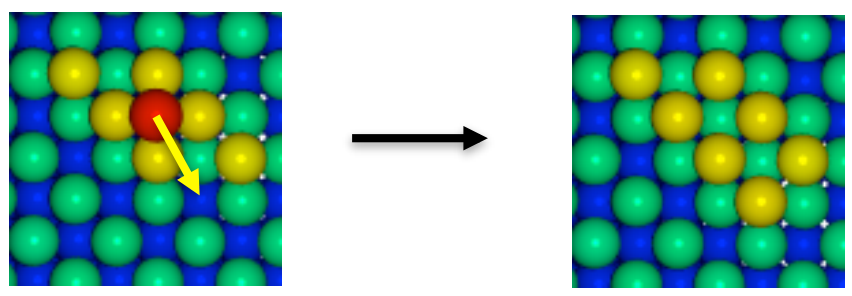

FIG. 13. (color online) The ES barrier was found to be identical to the $\{111\}$ surface at $0.42 \mathrm{eV}$ for an $\mathrm{Ag}$ atom to drop off the edge of a step. This barrier was rarely used, however, due to other transitions such as the small cluster formation and rearrangement requiring less energy to take place.

onto larger islands with step edge bonding barriers of around $0.03 \mathrm{eV}$. The reverse barrier; for single atoms

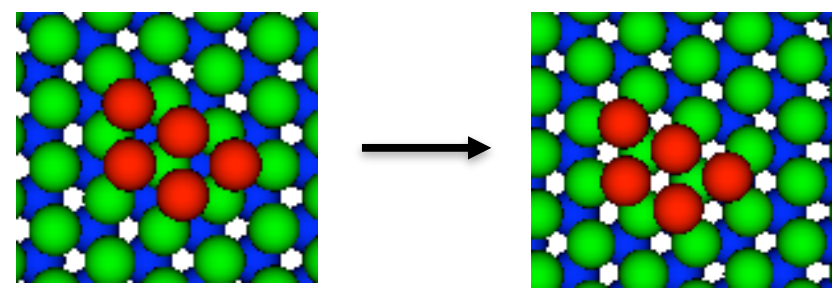

FIG. 14. (color online) A five atom cluster on the $\mathrm{Al}\{111\}$ surface which switches from $\mathrm{ABC}$ to $\mathrm{ABAB}$ stacking sequences with relatively low activation energy of $0.3 \mathrm{eV}$. During later steps of the simulation the cluster switches back to ABC with a lower barrier and then resulting growth continues in the correct stacking formation, perhaps by chance. Atoms are coloured by height of monolayer with red being the cluster on top. 


\begin{tabular}{cccc}
\hline \hline Monomer hop & 5-mer hop & Bond to step edge & De-bond from step edge \\
\hline$<0.1 \mathrm{eV}$ & $0.3 \mathrm{eV}$ & $0.03 \mathrm{eV}$ & $0.42 \mathrm{eV}$ \\
\hline \hline
\end{tabular}

TABLE III. Transition barriers for single ad-atom and small cluster diffusion on the $\mathrm{Al}\{111\}$ surface.

to de-bond from edges is huge in comparison. Another reason for the completeness of the evaporation growth here is illustrated in figure 14, planar clusters form during the simulation which are mobile up to five atoms, in this case requiring $0.3 \mathrm{eV}$ to diffuse. Therefore if large clusters are able to diffuse across the surface with accessible barriers this will aid the completion of layers as clusters have the ability to move to join one another.
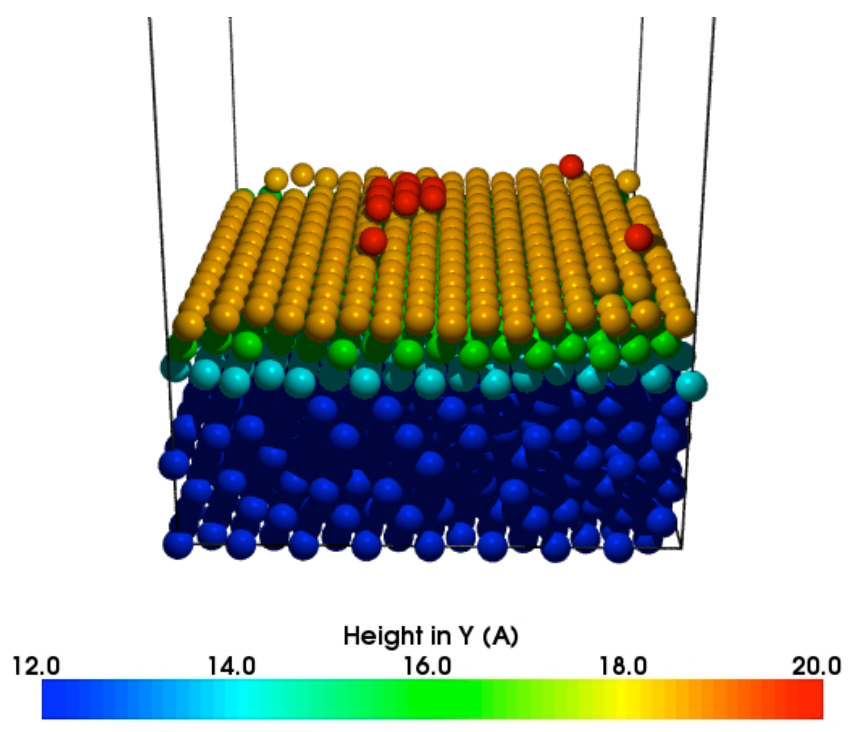

FIG. 15. (color online) Al $\{111\}$ growth via evaporation deposition after $0.29 \mathrm{~s}$ of real time. 672 atoms have been added to the system, equivalent to three complete monolayers, corresponding to a growth rate of ten monolayers per second. Two complete layers are formed, along with a third almost complete and a fourth just beginning. The original substrate was at $12 \AA$.

The most likely explanation for the completeness and crystallinity of the evaporation growth is however shown in figure 16. Illustrated is the ES barrier which has been calculated for $\mathrm{Al}\{111\}$ be only $0.07 \mathrm{eV}$. This tiny ES barrier plays a key role in the better quality of growth observed in figure 15 as small ES barriers allow atoms to drop off islands to complete the monolayer below, thus enabling growth via layer-by-layer completion rather than the cluster growth observed with Ag. Comparing $0.07 \mathrm{eV}$ with the $0.42 \mathrm{eV}$ ES barrier occuring with $\mathrm{Ag}$, this provides a convincing explanation of the different growth produced by these two metals.

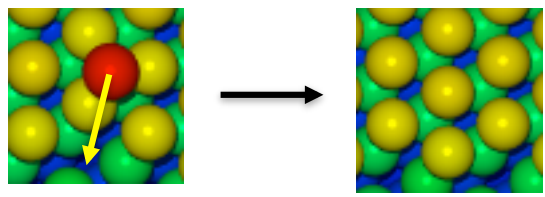

FIG. 16. (color online) The film completes via this ES barrier which allows layer-by-layer growth by atoms dropping off step edges with a very low barrier of $0.07 \mathrm{eV}$. The $\mathrm{Al}\{111\}$ grown via evaporation is clear evidence that this ES barrier plays an important role in the type of growth observed and hence the quality of the resulting film.

\section{Evaporation Deposition with Ion-Beam Assist}

Figure 17 shows thin film growth produced when an Ar ion-beam assist is used in conjunction with the evaporation method. Ar ions bombard the surface with $100 \mathrm{eV}$ of kinetic energy and strike the surface with equal probability as the depositing $\mathrm{Al}$ atoms. Ar bombardment has previously been found to increase the density of the material and promote surface diffusion ${ }^{5,8,9}$. Close inspection of figure 17 reveals that the Ar impacts have in fact severely damaged the new surface and the original substrate. The high energy of Ar bombardment allows penetration though three layers, displacing $\mathrm{Al}$ atoms from the substrate. $57 \mathrm{Ar}$ ions $(0.07 \%$ of the Ar bombarded throughout the simulation) have remained in the system, many of which have formed into subsurface clusters as reported in the literature ${ }^{10-13}$. Figure 18 illustrates the $\mathrm{Ar}$ agglomeration into subsurface clusters. Ar below the surface was found to diffuse freely through the substrate with energy barriers as low as $0.05 \mathrm{eV}$, similar to previous calculations on $\mathrm{Au}^{28}$. During the simulation some $\mathrm{Ar}$ does leave the system, usually after another Ar impact which transfers enough kinetic energy to the subsurface Ar, to allow some to escape from the substrate. The low ES barrier did aid this simulation also by allowing layers to complete and form around the Ar subsurface clusters, however these clusters have disrupted the growth so much that $\mathrm{Al}$ atoms are out of place.

\section{Sputter Deposition}

Figures 19 and 20 illustrate growth produced by magnetron sputtering, firstly ignoring the influence of $\mathrm{Ar}$ ions present in the magnetron plasma and then including their effect. When $\mathrm{Ar}$ is not included, new monolayers are almost complete in a similar fashion to the evaporation simulation results. Due to the higher 

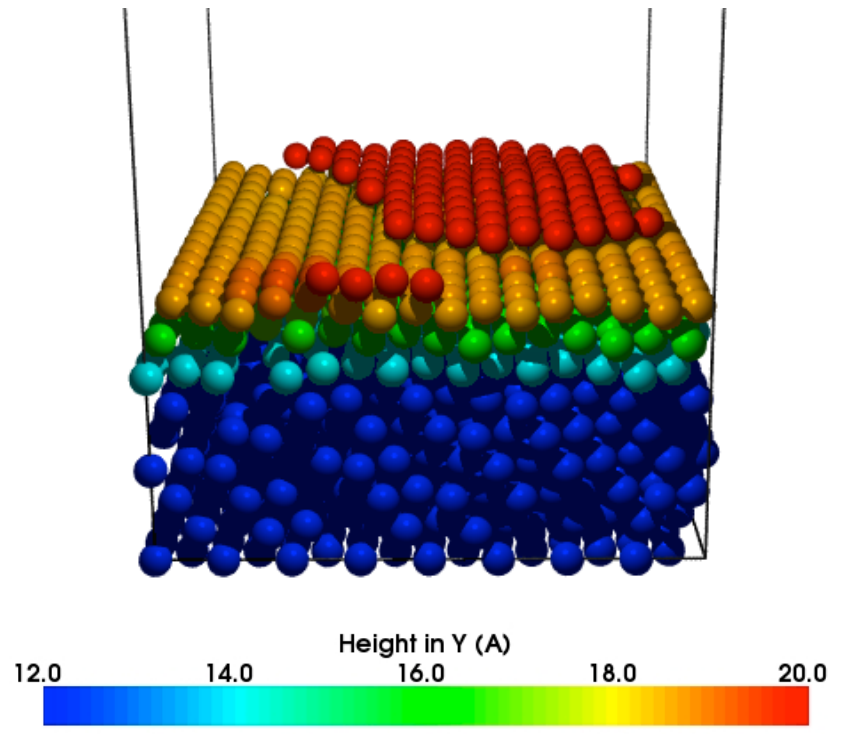

FIG. 17. (color online) Al $\{111\}$ growth via evaporation deposition with ion-assist, where Ar ions bombard the surface with $100 \mathrm{eV}$ of kinetic energy. Here $0.31 \mathrm{~s}$ of real time is simulated. From the addition of three monolayers of atoms, no complete monolayers have been formed. In fact, the original substrate itself is damaged from the high energy Ar impacts which penetrate up to three layers deep. Sub-surface Ar clusters have displaced $\mathrm{Al}$ atoms creating large $\mathrm{Al}$ voids in the substrate. Four new monolayers, all incomplete, are formed. Atoms are coloured by height in $\AA$.

energy deposition, mixing between the original substrate and any added atoms is more prominent than in the evaporation simulation which allows a damage and repair mechanism to take place which in turn allows increased surface diffusion and leads to complete layers. Illustrated in figure 20 is the resulting growth from the inclusion of the effect of $\mathrm{Ar}$ ions in the simulation. It is clear that Ar bombardment at $40 \mathrm{eV}$, transfers enough kinetic energy to the surface to allow almost perfect crystalline growth but unlike the $100 \mathrm{eV}$ model, the $\mathrm{Ar}$ has insufficient energy to penetrate the third layer and no Ar subsurface clusters are formed. Only 4 atoms are missing from the third layer, the rest being complete.

Comparing the resulting thin films, evaporation produces a complete film, whereas the addition of the ion-beam assist damages the surface beyond repair giving an incomplete, void filled structure with subsurface $\mathrm{Ar}$ clusters. Sputtering produces dense, crystalline and complete films, both with and without the inclusion of the effect of the Ar atoms in the plasma.

As observed with the $\mathrm{Ag}$, multiple atom concerted motions aid the growth to become complete by vacancy filling and the ES barrier where atoms drop off islands in order to complete the monolayer below. Figure 16 illustrates the ES barrier is very small for $\mathrm{Al}\{111\}(0.07 \mathrm{eV})$, suggesting that even with low energy evaporation depo- sition, atoms will very often drop off a step edge enabling layers to become complete. This was confirmed in the results of our evaporation simulation where growth was almost as complete as the sputter deposited growth. From the higher energy depositions observed during the sputtering process we notice damage and repair mechanisms allowing the slightly more complete growth. A concerted motion observed during island formation is depicted in figure 21, where two $\mathrm{Al}$ atoms slide across in unison to fill a vacancy within the island forming on the surface with a barrier of $0.25 \mathrm{eV}$.

\section{Deposition on the Al $\{100\}$ Surface}

Simulations are now repeated on the $\mathrm{Al}\{100\}$ surface. However, due to computational time limits $\mathrm{Ar}$ is not included in any of the following simulations. For these simulations it was noticed that energy barriers below 0.2 $\mathrm{eV}$ produced transitions that resulted in no net diffusion such as rotating trimers on the surface. By filtering out these low energy barriers we saved computational time, but at the possible expense of missing the occasional important transition. Table IV lists some of the main transitions observed during the growth simulation; the monomer hop and bonding and de-bonding from step edges.

\section{Deposition by Evaporation}

Figure 22 illustrates the growth of $\mathrm{Al}\{100\}$ from evaporation of $\mathrm{Al}$ onto the surface. From the addition of four monolayers of atoms to the system two monolayers are totally complete, with the third and fourth incomplete. Some mixing between atoms in the original substrate and new atoms is seen, however due to the low impact energy $\mathrm{Al}$ atoms tend to stick on the surface. The ES barrier shown in figure 23 has been calculated to be $0.24 \mathrm{eV}$, larger than on the $\{111\}$ surface but lower than $\mathrm{Ag}$ and low enough for the transitions to occur between deposition events.

\section{Sputter Deposition}

Figure 24 shows the grown film from sputtering $\mathrm{Al}$ onto the $\mathrm{Al}\{100\}$ surface. Almost all three new monolayers are complete, missing only two atoms from the third. The higher energy transferred from the sputtering, enabling greater surface diffusion, along with the accessible ES barrier, promote the completion of monolayers.

Comparing the resulting growth of $\mathrm{Al}\{100\}$ via evaporation deposition and sputtering, it is clear that both methods produce near-complete structures. Sputtering 


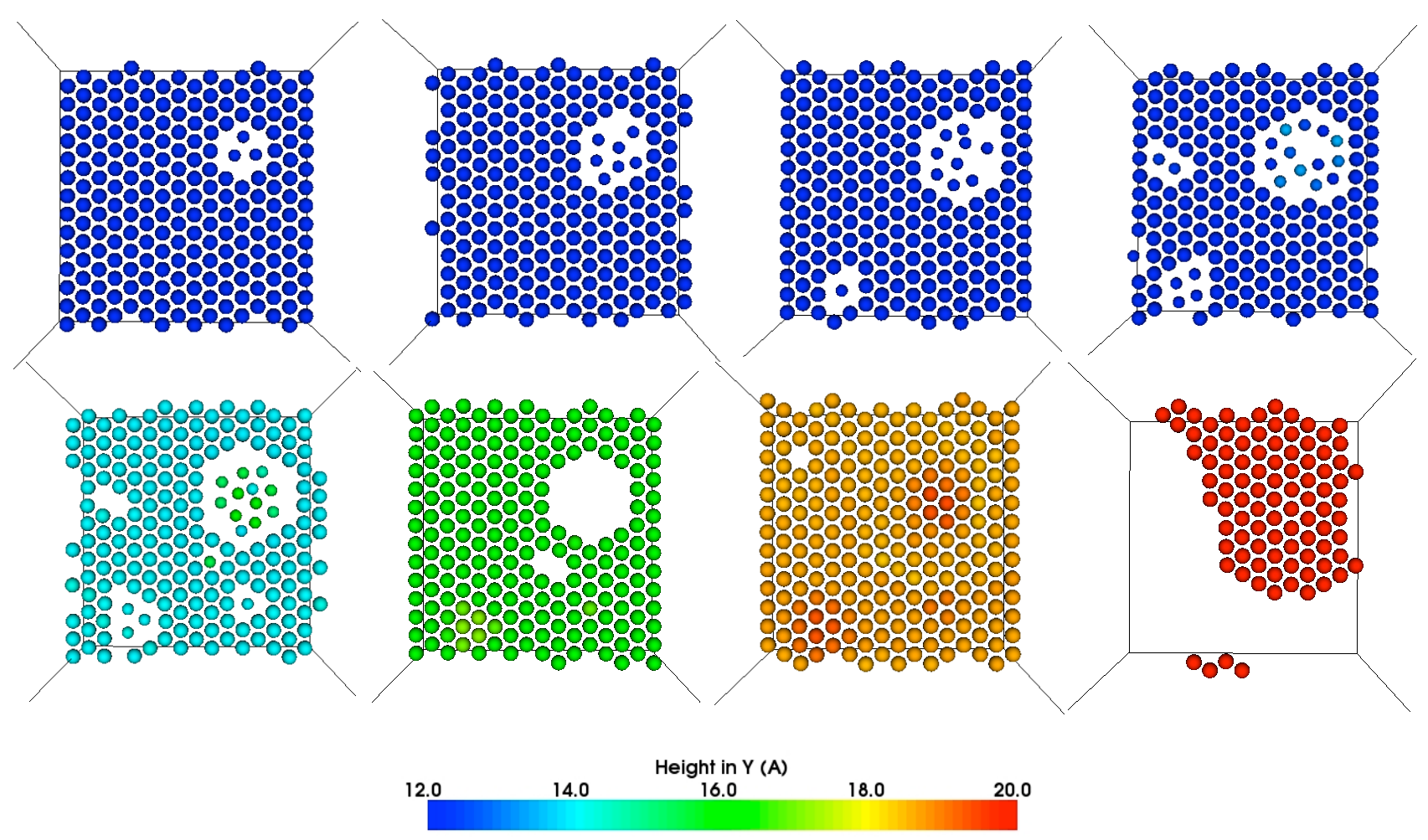

FIG. 18. (color online) Layers of $\mathrm{Al}\{111\}$ after evaporation growth with ion-beam assist. Layers are ordered left to right, beginning with the deepest layer and ending with the very top new layer. The newly grown layers are incomplete and Ar has created voids in the Al structure by sitting in subsurface Ar clusters. Al atoms are slightly larger diameter, with the Ar represented by smaller spheres.

\begin{tabular}{ccc}
\hline \hline Monomer hop via replacement mechanism & Bond to step edge & De-bond from step edge \\
\hline $0.27 \mathrm{eV}$ & $0.22 \mathrm{eV}$ & $0.51 \mathrm{eV}$ \\
\hline
\end{tabular}

TABLE IV. Transition barriers for single ad-atom and small cluster diffusion on the Al $\{100\}$ surface.

however produces a slightly more crystalline and complete film due to the increased kinetic energy transferred to the system from depositions.

The most prominent growth mechanism noticed during the $\mathrm{Al}\{100\}$ simulations is the two atom replacement mechanism where an atom diffuses over the surface via replacing a $\mathrm{Al}$ atom in the surface and pushing the original $\mathrm{Al}$ atom up onto the surface, occurring with an energy barrier of $0.27 \mathrm{eV}$, illustrated in figure 25. Shown in figure 23 is the ES barrier which requires $0.24 \mathrm{eV}$ to occur and enables the completion of the films even from the low energy evaporation. This is similar to what was observed with the $\{111\}$ surface.

\section{CONCLUSIONS}

Otf-KMC allows simulation of thin film growth over realistic time scales in comparison with traditional methods used; seconds of real time have been simulated. Another significant advantage of these methods is the ability to identify complicated growth mechanisms, some of which have not been studied at the atomistic level before. Understanding these complicated and often multiple atom transitions allows thin film growth to be understood to a much greater depth.

$\mathrm{Ag}\{111\}$ film growth was simulated for around $0.3 \mathrm{~s}$ real time allowing the deposition of three new monolayers onto the surface. Deposition by evaporation produced a clearly incomplete film full of vacancies and voids, with stacking faults occurring. The ion-beam assist promoted mixing with the original substrate and transferred kinetic energy to the system thus growth became slightly more complete. Sputtering however produced more dense, crystalline and complete films due to the increased kinetic energy within the system. The inclusion of the effect of Ar ions from the magnetron plasma did not change the resulting quality of the film, illustrating that the the $40 \mathrm{eV}$ from the $\mathrm{Ag}$ was enough energy by itself to promote diffusion and mixing. The calculation of barriers on the $\{111\}$ surface showed that the monomer hop required the least energy thus during 


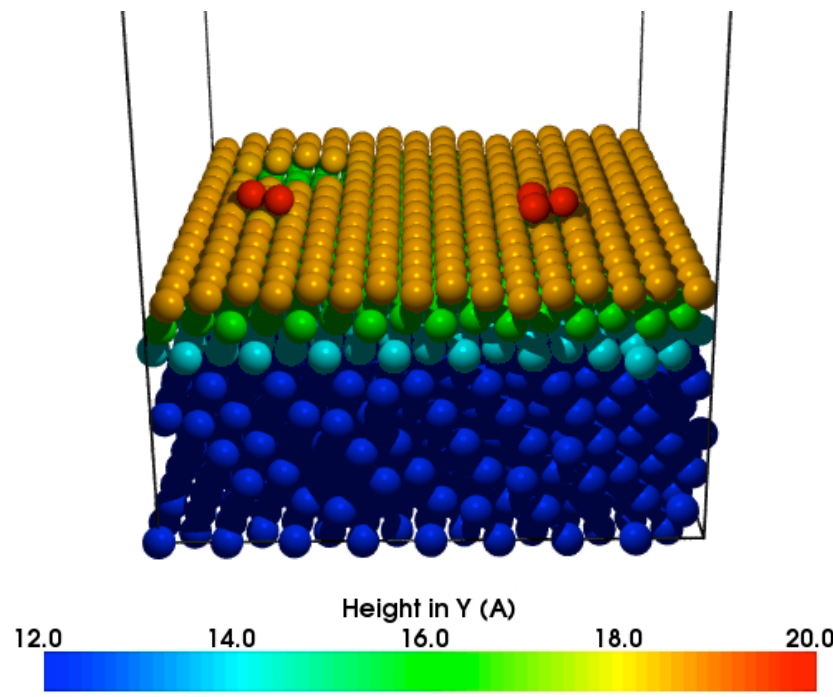

FIG. 19. (color online) Al $\{111\}$ thin film growth by magnetron sputter deposition after $0.3 \mathrm{~s}$ of real time. Three monolayers of $\mathrm{Al}$ are deposited onto the surface, and four new monolayers are created, two of which are complete and the fourth has only 5 atoms so is in very early stages of growth. Due to the higher energy impacts of Al than with evaporation deposition, some $\mathrm{Al}$ atoms penetrate the surface and can displace some atoms within the original substrate. This has resulted in very few atoms missing from the original substrate, producing a highly complete film. Atoms are coloured by height in $\AA$.
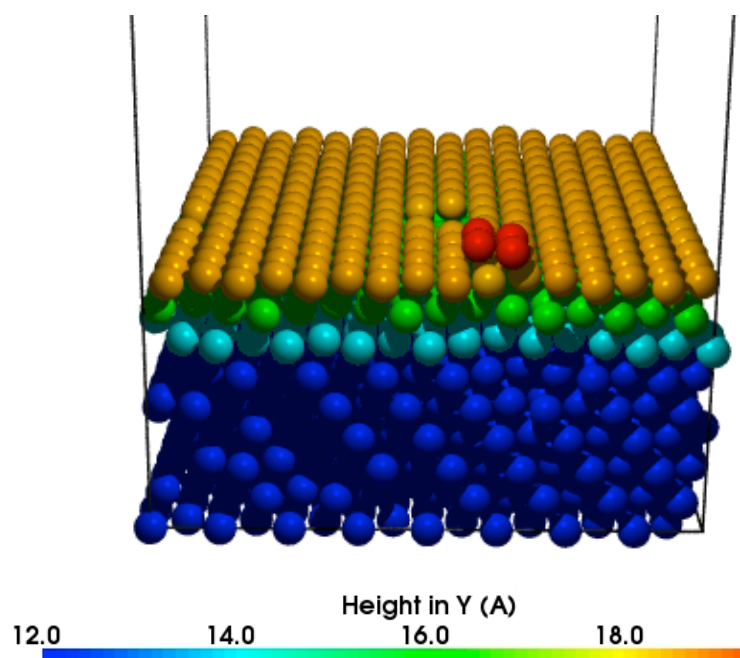

$$
14.0
$$$$
\text { Height in } Y(A)
$$$$
16.0
$$

18.0

FIG. 20. (color online) Al $\{111\}$ thin film growth via magnetron sputter deposition after $0.35 \mathrm{~s}$ of real time, including the effect of the Ar present in the plasma. After the deposition of three monolayers onto the surface, four new monolayers are created, two of which are complete and the third is only missing 4 atoms which are sit on top creating a fourth monolayer. Atoms are coloured by height in $\AA$.

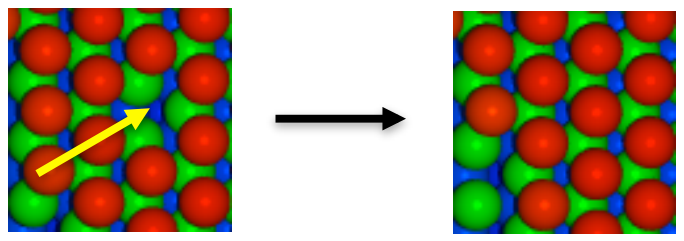

FIG. 21. (color online) An island formed on the $\mathrm{Al}\{111\}$ surface has a vacancy within it. Here two Al atoms slide across together in order to fill the vacancy. The energy barrier for this transition is $0.25 \mathrm{eV}$ which is highly accessible within the time frame, between successive ion impacts. Atoms are coloured by height.

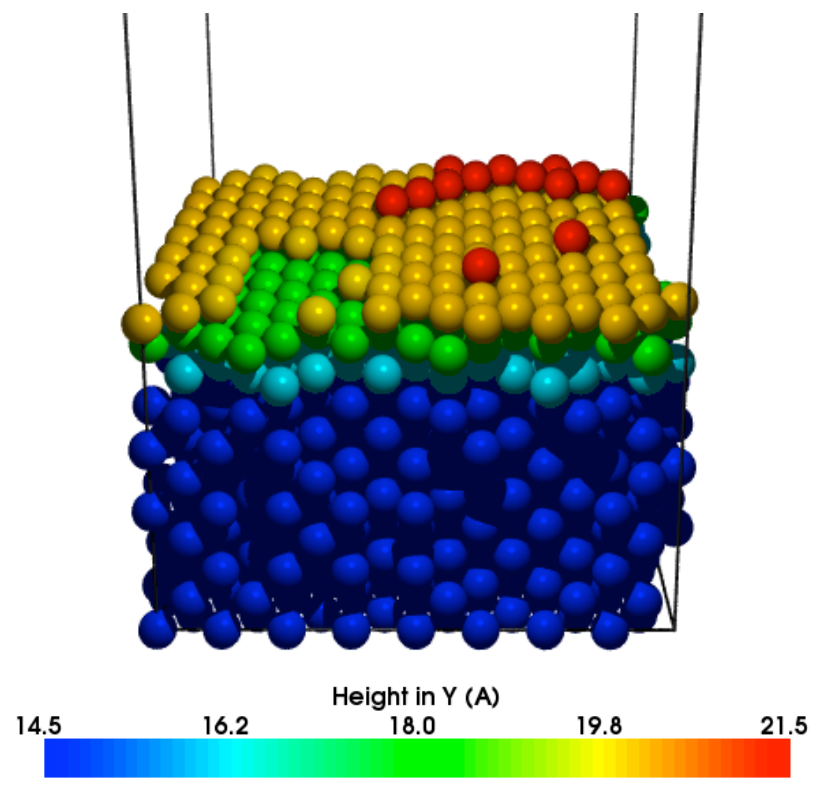

FIG. 22. (color online) Al $\{100\}$ growth from evaporation deposition simulated over $0.15 \mathrm{~s}$ of real time. 384 atoms are added to the system, equating to three complete monolayers. Four new monolayers are formed, two of which are complete. Atoms are coloured by height in $\AA$. The original substrate was at $14.5 \AA$.

all simulations it was this single ad-atom hop that was the most enabling single atom mechanism to produce clusters on the surface.

Thin film growth on the $\{100\}$ surface followed similar patterns; the evaporation process created highly incomplete monolayers with islands forming but no complete layers. Sputtering however, grew a much denser and more complete film with all new monolayers almost complete. Transitions observed during the growth simulations on the $\{100\}$ surface were predominately cluster formation and rearrangements as monomer hops required the most energy to occur. This meant that growth occurred via clusters on the surface growing and diffusing towards one another, creating larger clusters and islands.

A very important transition in enabling film growth is 


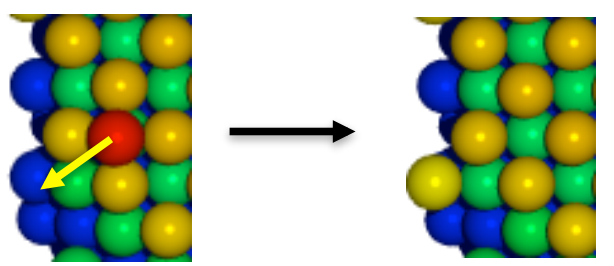

FIG. 23. (color online) The ES barrier for $\mathrm{Al}\{100\}$ is calculated to be $0.24 \mathrm{eV}$, low enough to occur between deposition events enabling layer-by-layer growth.
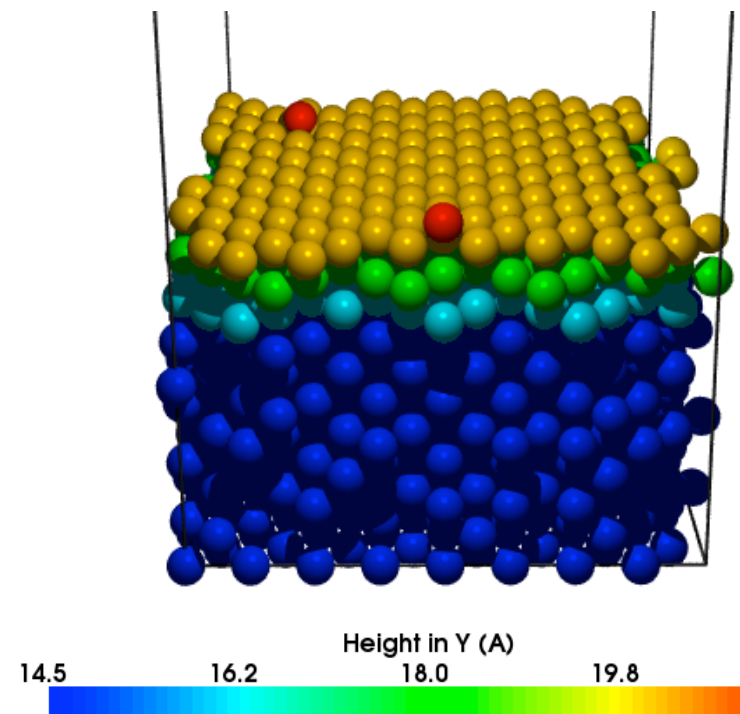

Height in $Y(A)$

14.5 16.2

18.0

19.8

FIG. 24. (color online) Al $\{100\}$ growth produced from magnetron sputtering over $0.18 \mathrm{~s}$ of real time. From three monolayers added, two complete monolayer are formed, with the third missing only two atoms, which we would expect would fill in and complete if the simulation was to continue. Atoms are coloured by height in $\AA$.

the ES (Ehrlich-Schwoebel) transition. On Ag surfaces the ES barrier was calculated to be $0.42 \mathrm{eV}$, which in comparison to other barriers available is high and therefore will rarely occur. Growth is thus seen to occur in a cluster-by-cluster way, as earlier illustrated in figure 9 , where atoms rarely drop down. Sputtering, however,

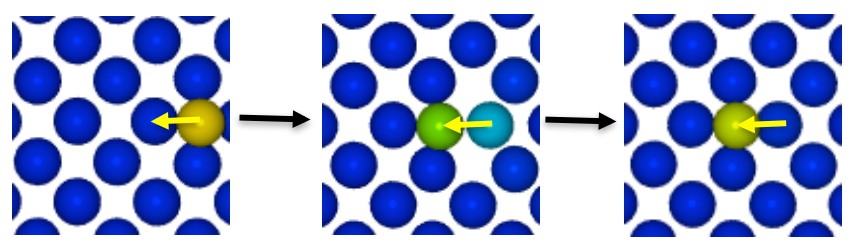

FIG. 25. (color online) $\mathrm{Al}\{100\}$ two atom concerted replacement mechanism is shown here in three steps; initial positions, saddle point and final positions. This mechanism is how single $\mathrm{Al}$ atoms diffuse around the surface with activation energy of $0.27 \mathrm{eV}$. Atoms are coloured by height, dark blue being the surface. transfers increased energy to the system which leads to increased disruption and atomic mixing enabling the films to be more complete.

Al $\{111\}$ films grown via evaporation and sputtering appear to be very similar to one another, contrary to expectation and to that observed with the Ag. Evaporation actually grew a close to complete film with two complete layers formed. The ES barrier was calculated as $0.07 \mathrm{eV}$, enabling the complete growth not usually seen from evaporation. Growth occurred in a layer-by-layer style, as earlier illustrated in figure 16, where atoms preferentially filled any vacancies in the layer below rather than beginning new clusters on the top.

When Ar was included via the ion-beam assist, results again were not as expected. The Ar assist, instead of aiding in densifying and completing the film as with $\mathrm{Ag}$, actually damaged the film beyond repair. Al voids were produced below the surface, enabling Ar subsurface clusters to sit. This subsurface Ar agglomeration observed agrees with results reported in the literature ${ }^{10-13}$. The Ar clusters destroy the substrate producing a highly incomplete, porous structure, however $\mathrm{Al}$ atoms continue to grow in the layer-by-layer style around the Ar.

Sputtering also produced highly complete films, with vacancies only in the top layer. The introduction of increased kinetic energy to the system from the higher energy Al depositions enabled increased surface diffusion. When the effect of the Ar in the plasma was included, results improved very slightly due to the increase in energy transferred from the Ar bombardments.

The $\{100\}$ surface showed similar results to the previous surface. Evaporation growth produced rather complete monolayers with many vacancies, but only in the top layer. The ES energy barrier on this surface was calculated as $0.24 \mathrm{eV}$, lower than the energy barrier for a single ad-atom diffusing over the surface (this required $0.27 \mathrm{eV})$. Although the ES barrier is not as low as on the $\{111\}$ surface, it is still accountable for the good quality of the growth produced in comparison to the Ag evaporation growth. Higher energy depositions in sputtering produced highly complete and dense film with all three new monolayers grown perfectly with only two vacancies in the top layer, a notable improvement on the evaporation growth.

Otf-KMC has allowed a much deeper understanding of the process of thin film growth and the mechanisms involved, some of which are non-intuitive. It is clear from this work that the energetics of the deposition process utilised plays a significant role on the surface morphology and the film quality, and that $\mathrm{Ag}$ and $\mathrm{Al}$, although both simple structured FCC metals, actually grow very differently due to differences in barriers for 
important transitions.

\section{ACKNOWLEDGEMENTS}

The authors would like to thank EPSRC and TSB for financial support and Loughborough University for the
High Performance Computing time. One of the authors (S. Blackwell) thanks Loughborough University for a postgraduate studentship. This work was funded by EPSRC grant no. EP/C524322/1.
1 M. Brogren, P. Nostell, and B. Karlsson, Sol. Energy 69, 173 (2001).

2 J. S. Coventry, Sol. Energy 78, 211 (2005).

3 D. A. Jaworske, in Energy Conversion Engineering Conference, 1997. IECEC-97., Proceedings of the 32nd Intersociety, Vol. 1 (1997) pp. 407-411.

4 J. T. Cheung and H. Sankur, Crit. Rev. Solid State Mater. Sci. 15, 63 (1988).

${ }^{5}$ F. A. Smidt, Int. Mat. Rev. 35, 61 (1990).

${ }^{6}$ I. Safi, Surf. Coat. Tech. 127, 203 (2000).

7 K. Reichelt and X. Jiang, Thin Solid Films 191, 91 (1990).

8 R. D. Bland, G. J. Kominiak, and D. M. Mattox, J. Vac. Sci. Technol. 11, 671 (1974).

9 D. M. Mattox and G. J. Kominiak, J. Vac. Sci. Technol. 9, 528 (1972).

10 R. S. Dhaka, K. Gururaj, S. Abhaya, G. Amarendra, S. Amirthapandian, B. K. Panigrahi, K. G. M. Nair, N. P. Lalla, and S. R. Barman, J. Appl. Phys. 105, 054304 (2009).

11 C. J. Rossouw and S. E. Donnelly, Phys. Rev. Lett. 55, 2960 (1985).

12 C. Biswas, A. K. Shukla, S. Banik, S. R. Barman, and A. Chakrabarti, Phys. Rev. Lett. 92, 115506 (2004).

13 M. Schmid, W. Hebenstreit, P. Varga, and S. Crampin, Phys. Rev. Lett. 76, 2298 (1996).

14 V. Georgieva, A. F. Voter, and A. Bogaerts, Cryst. Growth Des. 11, 2553 (2011).

15 G. Hu, G. Orkoulas, and P. D. Christofides, in American Control Conference (ACC '09) (2009) pp. 4797-4804.

16 B. F. Gordiets, J. L. Andujar, C. Corbella, and E. Bertran, Euro. Phys. J. D 35, 505 (2005).

17 S. Blackwell, R. Smith, S. D. Kenny, L. J. Vernon, and J. M. Walls, J. Mat. Res. 27, 799 (2012).

18 M. R. Sorensen and A. F. Voter, J. Chem. Phys. 112, 9599 (2000)

19 C. L. Liu, J. M. Cohen, J. B. Adams, and A. F. Voter, Surf. Sci. 253, 334 (1991).

20 A. F. Voter, Phys. Rev. Lett. 78, 3908 (1997).

21 S. Xiao and W. Hu, J. Cryst. Growth 286, 512 (2006).

${ }^{22}$ G. Henkelman and H. Jónsson, Phys. Rev. Lett. 90, 116101 (2003).
23 M. Li, Y. Han, P. A. Thiel, and J. W. Evans, J. Phys.: Condens. Matter 21, 084216 (2009).

24 L. J. Vernon, Ph.D. thesis, Loughborough University (2010).

25 A. F. Voter, F. Montalenti, and T. C. Germann, Annu. Rev. Mat. Res. 32, 321 (2002).

26 A. F. Voter, "Radiation effects in solids," (Springer, NATO Publishing Unit, Dordrecht, The Netherlands, 2005).

27 G. Henkelman and H. Jónsson, J. Chem. Phys. 115, 9657 (2000).

28 C. Scott, S. Blackwell, L. Vernon, S. D. Kenny, J. M. Walls, and R. Smith, J. Chem. Phys. 135, 174706 (2011).

29 G. J. Ackland, D. J. Bacon, A. F. Calder, and T. Harry, Philos. Mag. A 75, 713 (1997).

30 A. F. Voter and S. Chen, in Characterization of Defects in Materials, edited by R. W. Siegel and et al (1978) pp. $175-180$.

31 N. W. Ashcroft and D. N. Mermin, "Solid state physics," (Ashcroft, N. W. and Mermin, D. N., 1976) Chap. 20: Cohesive Energy, pp. 395-414.

32 J. F. Ziegler, J. P. Beirsack, and U. Littmark, The stopping and range of ions in solids, Vol. 1 (Pergamon, New York, 1985).

33 H. J. C. Berendsen, J. P. M. Postma, W. F. van Gunsteren, A. DiNola, and J. R. Haak, J. Chem. Phys. 81, 3684 (1984).

34 G. Henkelman, B. P. Uberuaga, and H. Jónsson, J. Chem. Phys. 113, 9901 (2000).

35 G. Henkelman and H. Jónsson, J. Chem. Phys. 113, 9978 (2000).

36 G. Henkelman, D. Sheppard, and R. Terrell, J. Chem. Phys. 128, 1 (2008).

37 G. Vineyard, J. Phys. Chem. Solids 3, 121 (1957).

38 C. J. Howe, M. D. Cropper, R. M. Wardle, P. Bailey, and T. C. Q. Noakes, Surf. Sci. 604, 1658 (2010).

39 R. L. Schwoebel and E. J. Shipsey, J. Appl. Phys. 37, 3682 (1966).

40 G. Ehrlich and F. G. Hudda, J. Chem. Phys. 44, 1039 (1966). 